\title{
ПОСТРОЕНИЕ СЕЙСМИЧЕСКИХ ИЗОБРАЖЕНИЙ В ИСТИННЫХ АМПЛИТУДАХ С ИСПОЛЬЗОВАНИЕМ ГАУССОВЫХ ПУЧКОВ ПО ДАННЫМ ВСП
}

Протасов М.И., Чеверда В.А.

(ИНГГ СО РАН, г.Новосибирск, Россия)

В данной работе представлен оригинальный подход построения сейсмических изображений в истинных амплитудах, основанный на взвешенном суммировании данных ВСП. Для вычисления весов требуется трассирование пары гауссовых пучков из текушей точки целевой области в направлении системы наблюдения. Сиециальный выбор гауссовых пучков позволяет учесть геометрическое расхождение и условия освещения в конкретной системе наблюдения. Более того, глобальная регулярность гауссовых пучков даёг возможность работать в произвольном поле лучей.

Скорость распространения волн представляется в виде суперпозиции 'двух составляющих: $c_{0}(x, z)$ - гладкая, плавно меняющаяся макроскоростная комтонента, напротив $c_{1}(x, z)$ описывает локальную, быстро осциллирующую компоненту. Для описания рассеянных/отраженных волн $u_{1}\left(x, z ; x_{s} ; \omega\right)$ используется Борновское приближение. Требуется восстановить локальную изменчивость скоростного строения $\frac{c_{1}(x, z)}{c_{0}(x, z)}$ по известньм в скважине $\quad x=0$ данным ВСП: $\varphi\left(z_{r}, x_{s}, \omega\right)=u_{1}\left(0, z_{r} ; x_{s} ; \omega\right)$ (1). Зафиксируем некоторую текущую точку $\left(x_{i}, z_{i}\right)$, принадлежапую целевой области $D$ и выпустим из нее пару лучей: один в направлении свободной поверхности $z=0$, другой в направлении линии расположения приемников (в направлении скважины) $x=0$, вдоль них построим пару гауссовых пучков [4]. Эти лучи полностью определяются заданной макроскоростной моделью, а таюже их углом наклона $\alpha$ и углом раствора $\beta$ (см. Рис. 1). Двойное использование теоремы Грина приводит к следующему соотнопению:

$$
\begin{aligned}
& 2 \omega^{2} \iint_{X} \frac{1}{c_{0}^{2}(\xi, \eta)} \frac{c_{1}(\xi, \eta)}{c_{0}(\xi, \eta)} u^{(g b)}\left(\xi, \eta ; x_{i}, z_{i} ; \alpha, \beta ; \omega\right) u_{i}^{(\xi)) \times} \\
& \times\left(\xi, \eta ; x_{i}, z_{i} ; \alpha, \beta ; \omega\right) d \xi d \eta= \\
& =\left.\int_{x=0} \int_{x=0}^{\left.\frac{\partial u_{s}^{(g)}\left(x_{r}, z ; x_{i} ; z_{i} ; \alpha, \beta ; \omega\right)}{\partial z}\right|_{z=0} \times} \frac{\partial u_{r}^{(s))}\left(x, z_{r} ; x_{i}, z_{i} ; \alpha, \beta ; \omega\right)}{\partial x}\right|_{s=0} \varphi\left(z_{r}, x_{s} ; \omega\right) d x_{s} d z_{r}
\end{aligned}
$$

Далее умножим обе части соотнопения (2) на $\exp \left\{-i \omega\left(\tau_{r}\left(x_{i}, z_{i}\right)+\tau_{s}\left(x_{i}, z_{i}\right)\right)\right\} \frac{2 \cos ^{2} \beta}{\omega}$, проинтегрировав затем по временной частоте $\omega$ и углу наклона $\alpha$. Введём замену переменных $p_{x}=\frac{2 \omega \cos \beta}{c_{0}\left(x_{i}, z_{i}\right)} \sin \alpha ; \quad p_{z}=\frac{2 \omega \cos \beta}{c_{0}\left(x_{i}, z_{i}\right)} \cos \alpha$. Далее непосредственными вычислениями получаем главное значение высокочастотного разложения «функщии изображения" в текущей точке:

Как видно, правая часть выраженни (3) является суперпозицией двух линейных интегральных

$$
\Phi\left(x_{1}, z_{i}\right)=\iiint_{x_{j \neq}(x, y)} d p_{1} d p_{x} \iint_{r(3, \eta)} \frac{c_{1}(\xi, \eta)}{c_{0}(\xi, \eta)} \times \exp \left(i p_{\mathrm{x}}\left(x_{i}-\xi\right)+i p_{\mathrm{z}}\left(z_{1}-\eta\right)\right) d \xi d \eta
$$

операторов - преобразования Фурье по пространственным переменным от искомого возмущения макроскоростной модели $\frac{c_{1}(x, z)}{c_{0}(x, z)}$ и "непопного" обратного преобразования Фурье. Мы называем обратное преобразование «неполным», потому что множество в спектральной плоскости, по которому оно выполняется, является круговым сектором.

Дпя тестирования описанного выше метода построения изображений была взята синтетическая модель SIGSBEE2A, распространяемая совместным предприятием SMAART. Получённые численные результаты демонстрирует весьма высокое качество восстановления геометрического строения среды.

Предложенный в настоящей работе метод построения изображений в истинньх амплитудах в конечном итоге сводится к взвешенному суммированию исходных данных ВСП. Его основным отличием является использование для построения весов Гауссовых пучков, выпускаемых из целевой области в направлении системы наблюдения. Хочется также обратить внимание на чрезвычайно важное свойство изложенного подхода, позволяющее получать селективные изображения, содержащие только лишь заданным образом ориентированыые отражающие элементы. На этой основе может быть получена информация о распределении в среде неоднородностей субсейсмидеского масштаба.

Данная работа была выполнена при поддержке Schlumberger Moscow Research, и при частичной поддержке РФФИ проекты № 07-05-00538, № 05-0564277-а и гранта им. М.А. Лаврентьева для молодых ученьх СО РАН.

\section{ЛНТЕРАТУРА}

G. Beylkin, 1985. Imaging of discontinuities in the inverse scattering problem by inversion of a causal generalized Radon transform: Journal of Mathematical Physics, 26, $99-108$.

M.M.Popov, 2002. Ray theory and Gaussian beam for geophysicists EDUFBA, SALVADOR-BAHIA

Протасов М.И., Чеверда В.А. Построение сейсмических изображений в истинных амплитудах.// Доклады Российской Академки Наук, 2006, т.407(4), с. 441-446. 